Objectives To work collaboratively with senior researchers to develop compelling cases for actions that could be taken to address the most significant gaps between research evidence and health policy/practice in Australia.

Methods Faculty members will search literature, consult with stakeholder networks and debate issues in developing a paper of published evidence, recommending actions to address each prioritised gap and providing the rationale for prioritisation. Steering Groups will oversee the development of each Case for Action.

Results This presentation will share the experiences and lessons learnt to-date in developing Cases for Action.

Discussion There is a gap between what we know and what we do. Cases for Action will draw on the combined expertise of researchers to systematically consider and prioritise actions to best address these gaps. Possible actions that could be proposed include advice to government about health policy, clinical or public health guidelines, or opportunities to collaborate with strategic partners to leverage investment in health or to provide support in the implementation of heath strategies.

Implications for Guideline Developers/Users The lessons learnt from the Cases for Action process will benefit attendees who are considering how to focus their effort to ensure that healthcare policy and practice best reflects available evidence.

\section{CAN HEALTH CARE NETWORKS DEVELOP AUTONOMY OVER DEVELOPMENT AND IMPLEMENTATION OF GUIDANCE WITHIN AN ENVIRONMENT SHAPED BY ACCREDITATION STANDARDS?}

S Goldman, M Koster, J Schottinger. Kaiser Permanente, Southern California Permanente Medical Group, Pasadena, USA

\section{0:1136/bmjgs-2013-002293.45}

Background Standards for clinical practice enacted by external accreditation organisations can limit the ability of health care organisations to develop and implement evidence-based guidance to improve clinical practice and health system efficiency, and reduce unnecessary testing.

Context As part of a system-wide effort to improve patient quality and access, medical specialists in a large group practice sought to determine whether standard bilateral venous duplex ultrasound (VDUS) scans were medically necessary in patients with unilateral signs and symptoms of deep vein thrombosis (DVT). Typically these patients receive bilateral exams; however, the high number of negative test results in non-symptomatic legs suggested bilateral testing may not be necessary.

Description of Best Practice An evidence review was conducted to evaluate whether unilateral VDUS scanning accurately identifies patients who can safely undergo unilateral VDUS exams in the symptomatic limb without missing a DVT in the unscanned, asymptomatic limb. The evidence review concluded that the number of undetected DVTs in the unscreened asymptomatic limb was very low, suggesting that unilateral VDUS screening in lower-risk patients (i.e., outpatients and patients without malignancy) could be safely performed. Accreditation standards, however, require bilateral screening in all patients, regardless of DVT risk status.

Lessons for Guideline Developers, Adaptors, Implementers, and/or Users Accreditation standards can hinder practice change and limit research for more effective and efficient practices. Some accrediting organisations accept feedback and adjust standards as new data emerges. Providing evidence-based information to these organisations may initiate changes in standards.

\section{TRACKING THE IMPACT OF THE AGENCY FOR HEALTHCARE RESEARCH AND QUALITY'S (AHRQ) EFFECTIVE HEALTH CARE PROGRAM THROUGH UPTAKE BY CLINICAL PRACTICE GUIDELINE AND QUALITY MEASURE DEVELOPERS}

${ }^{1} \mathrm{E}$ Erinoff, ${ }^{1} \mathrm{~V}$ Coates, ${ }^{2} \mathrm{M}$ Nix. ${ }^{1} \mathrm{ECRI}$ Institute, Plymouth Meeting, USA; ${ }^{2}$ Agency for Healthcare Research and Quality (AHRQ), Rockville, USA

\section{0:1136/bmjqs-2013-002293.46}

Background Public and private funders evaluate health care investments in terms of outcomes and accountability. Citation analysis can approximate the dissemination and impact of funded research outputs.

Objectives Use references in guidelines and measures represented in the National Guideline Clearinghouse (NGC) and National Quality Measures Clearinghouse (NQMC) to track the uptake of AHRQ Effective Health Care (EHC) programme outputs.

Methods 442 EHC-related titles were searched against the fulltext corpus of the Clearinghouses. Documents that cited the titles were examined for the context of the citations. References were considered strong when tied to a specific metric or recommendation or noted as important to the guideline's methodology; moderate if discussed in the body of the citing document; and weak if they appeared only in the reference list.

Results 174 individual guidelines and measures cited EHCrelated titles $(n=341) .50 \%$ of the guideline references were strong, $28 \%$ moderate and the remainder weak or undetermined (22\%). All measure references were strong.

Discussion This analysis has been done annually since 2010 with the numbers of detected citations increasing each year. The method used not only assesses whether a work was referenced in a guideline or measure, but its relative importance to the guideline or measure providing evidence of impact of the EHC programme.

Implications Systematic reviews and other research published through the EHC programme are being used to develop guidelines and measures that meet inclusion criteria for NGC and NQMC. EHC reports may be downloaded and topics nominated at www.effectivehealthcare.ahrq.gov

\section{RETIREMENT OF PERFORMANCE MEASURES IN A NATIONAL PAY FOR PERFORMANCE (P4P) SCHEME}

${ }^{1} \mathrm{D}$ Sutcliffe, ${ }^{1} \mathrm{~L}$ Hobbs, ${ }^{1} \mathrm{G}$ Flatt, ${ }^{1} \mathrm{E}$ Shaw, ${ }^{1,2} \mathrm{~T}$ Stokes, ${ }^{1} \mathrm{~N}$ Baillie. ${ }^{1}$ National Institute for Health and Care Excellence, Manchester, UK; ${ }^{2}$ University of Birmingham, Birmingham, UK

\section{0:1136/bmjqs-2013-002293.47}

Background P4P schemes, providing financial incentives across a range of improvement indicators, are widely used and can improve health outcomes. These systems can work at different levels, including at the national level. It is important that performance measures (PMs) used in such systems have a robust and up-to-date evidence base to support continued use and they remain fit for purpose; this involves selecting PMs for 'retirement'.

Objectives To: i) describe methods used in selecting PMs for retirement, ii) present alternative methods for selecting PMs for retirement. 
Methods Identifying PMs for retirement have been based on several methods including: i) reported trends in achievement and exception reporting ii) review of paired PMs iii) review of supporting evidence and/or technical specifications iv) modified Delphi.

Results We will present results of using these methods, and discuss alternatives to these (e.g., the Nominal Group Technique), and implications for retirement of PMs.

Discussion These methods have been successful in identifying indicators for retirement. To ensure continual improvements in quality of care delivered through the P4P scheme and provide opportunities for new areas to be added, the review and retirement of PMs remains important.

Implications for Guideline Developers/Users Guideline developers should be aware of key PMs based on guidance recommendations and have systems to ensure that underpinning evidence is up-to-date. PM developers should have processes to ensure that PMs are based on up-to-date evidence and remain fit for purpose.

\section{GUIDELINE BASED PERFORMANCE MEASURES TOWARDS G-I-N STANDARDS}

${ }^{1} \mathrm{M}$ Nothacker, ${ }^{2} \mathrm{~T}$ B Shaw, ${ }^{2,3} \mathrm{~T}$ Stokes. ${ }^{1}$ Association of the Medical Scientific Societies in Germany (AWMF), Berlin, Germany; ${ }^{2}$ Nationale Institute for Health and Clinical Excellence (NICE), Manchester, UK; ${ }^{3}$ University of Birmingham, Birmingham, UK

\section{0:1136/bmjqs-2013-002293.48}

Background Quality improvement in health care requires the development and use of performance measures (PMs) that address health care processes, outcomes and patient perspectives. PMs are increasingly being developed explicitly from clinical guideline (CG) recommendations. There are no agreed international standards for the development of guidelines based PM. The development of such standards has been agreed by the G-IN-PM Working Group (PMWG).

Objectives To develop a core set of standards for guidelines based PMs.

Methods - Systematic literature review of PM development methods - Identification of core components of guidelines based PM development - Development of draft standards for each core component - DELPHI process (at least 2 rounds) within the PMWG group to develop final set of standards

Results Essential components identified are: CG selection, extraction of CG recommendations, development of PMs from CG recommendations, assessment of potential PMs, intended use of PMs, piloting and review of PMs. The final agreed standards will be presented.

Discussion These guideline based PM standards will be refined and validated in future G-I-N PMWG projects.

Implications for Guideline Developers/Users This set of core standards for guideline based PM development offers guidance for PM developers on consensus based good practice. The resulting PM development process may also guide guideline developers to formulate more specific and measurable CG recommendations.

\section{THE IMPLEMENTATION FIELD TEAM 6 YEARS ON: APPROACHES TO ENGAGEMENT AND EVALUATING IMPACT}

J Moore, V Moore. National Institute for Health and Clinical Excellence, Manchester, UK

10:1136/bmjqs-2013-002293.49
Background The Implementation Field Team for this national guidance producing organisation has been established for six years. Seven consultants visit around 800 organisations annually, providing updates on national guidance, sharing examples of implementing good practice, and collecting feedback on our national guidance and barriers to implementation.

Context We have consistently evaluated our activities, but have found inherent difficulties with identifying impact, and have relied on proxy measures of success. As a new system for commissioning health services develops, we reviewed evidence around effective implementation activities and evaluating their impact. This led to innovative approaches to engagement and improved methods of evaluating impact.

Description of Best Practice We revised field team implementation strategies and activities to fit better with the new system of health commissioning. We conducted our own small scale survey, and also invited an external organisation to conduct a larger survey with field team clients to evaluate impact and to inform the planning and delivery of services in the future. We have moved from proxy measures of effectiveness evaluated every six months to newly developed "success criteria", which are outcomes focused, owned by the whole organisation, and identify three year incremental objectives for external engagement. This informs operational plans for future engagement activities.

Lessons for Guideline Developers, Adaptors, Implementers, and/or Users Evaluating the impact of implementation activities and teams is difficult but important and achievable. Focusing on immediate and intermediate implementation outcomes over longer timescales, and developing success criteria for field team implementation and engagement activities is valuable.

\section{SUCCESSFUL IMPLEMENTATION OF CARDIOVASCULAR PREVENTION AND TREATMENT GUIDELINES IN AN INTEGRATED HEALTH CARE SYSTEM: STRUCTURE, PROCESS AND OUTCOMES}

1J Harris, ${ }^{2} \mathrm{M}$ Jaffe. 'The Permanente Medical Group, San Rafael, USA; ${ }^{2}$ The Permanente Medical Group, South San Francisco, USA

\section{0:1136/bmjqs-2013-002293.50}

Background Evidence based guideline recommendations can support effective prevention and treatment of cardiovascular disorders, leading causes of morbidity and mortality in our population. Improvements result if recommendations are implemented in a uniform and effective manner.

Objectives To describe the process, structure and results of efforts to better manage cardiovascular risks and events in an integrated health system using organisational best practices; To present results of risk reduction, disease management and acute care programmes.

Methods Database analyses revealed opportunities for improvement. Pilot projects were conducted, followed by training about successful processes and practices, supported by organisational leaders. Ongoing comparative feedback supports improvements. Suggested order sets are incorporated in the EMR. Financial incentives for meeting targets accrue to medical centres.

Results The incidence of acute myocardial infarctions dropped significantly in the last 5 years, as did the mortality rate. Stroke mortality dropped significantly as well. The population levels of lipids, blood sugar, blood pressure and CHF control continue to improve.

Discussion Guideline recommendations were adopted across our delivery system when supported by top leadership, testing, 